\title{
Density of State (DOS) for Zig-Zag, Chiral and Armchair SWCNTs and DWBNNTs: In Viewpoint of Electronics and Structure
}

\author{
ZEYNAB NOURIMAJD \\ Department of Chemistry, Science and Research Branch, Islamic Azad University, Tehran, Iran \\ *Corresponding author E-mail: r.nourimajd@yahoo.com \\ http://dx.doi.org/10.13005/ojc/320223
}

(Received: March 10, 2016; Accepted: April 17, 2016)

\begin{abstract}
In this work we have simulated our system based on the various distribution diameters of SWCNTs, SWBNNTs and DWCNTs in three forms of zigzag, armchair and chiral. We have start for answering to some question about the mechanical, electronical and thermochemical properties of the diameter distribution on the various nanotubes, band gape, and potential difference between two layers of a nano cylindrical and finally the radial charge distribution of those systems. It is found that the energy gaps and the energy of those SWBNNTs are strongly dependent on their Chirality and diameters. The energy gap of the formed double-walled (DW-C\&BN-NTs ) can even be much reduced due to the coupled effect of wall buckling difference and inter-wall $\pi$ - $\pi^{\star}$ hybridization.
\end{abstract}

Keyword: SWCNTs, SWBNNTs, Zig-Zag, Chiral and Armchair.

\section{INTRODUCTION}

Control and designing of nanotube diameter is needed for developing nanotube growth methods. Nanotubes with a diameter of less than 10 angstrom provide the ideal nano space in one-dimension. Twenty years ago, it was been suggested that the size of the growth catalyst used in metal ${ }^{1}$ catalyzed chemical vapor deposition (CVD) can define the diameter of as-grown carbon nanotubes ${ }^{2}$. This idea has been confirmed by the observation that catalytic particles at the ends of CVD-grown nanotubes have sizes proportionate with the nanotubes diameters ${ }^{3}$.

Small-diameter carbon nanotubes exhibit many exotic properties such as anisotropic optical absorption spectra ${ }^{4}$ and superconductivity originated from a Peierls distortion ${ }^{5}$.

These findings have stimulated much interest in study of small nanotubes both experimentally and theoretically. 
Boron nitride nanotube (BNNT), which firstly predicted and synthesized by Rubio and Chopra respectively6, has a structural analogy to CNT but, contrary to the CNT being metallic (semiconductor) depending on chirality. BNNTs are usually can be an insulator regardless of their helicity, tube diameters and number of tube walls?

Besides, the study on double-walled BNNTs (DBNNTs) has demonstrated an interesting variation in their electronic properties when compared with those of freestanding component of BNNTs ${ }^{8}$. So it is also important to see the inter-wall coupling behavior and interaction energies associated with the small BNNTs.

Boron nitride nanotubes possess a large band gap $[\sim(5-6) \mathrm{eV})]$ irrespective of the number of walls, diameters, electronic properties and chirality ${ }^{9}$ In addition, they are so stable in viewpoint of chemical and mechanical structure ${ }^{10}$. Therefore, thin single-wall BNNTs (SWBNNs) can widely be used as an ideal nanotube for nano science to produce special materials such as atomic wires, capacitors and semiconductors. In our study the SWBNNs is a suitable material as an insulator to produce nanocylindrical capacitors.

They used arc-grown SWCNTs (with a diameter distribution of $1.4 \pm 0.1 \mathrm{~nm}$ ) as a template to synthesize thin SWBNNTs. The uniform diameter distribution of SWCNT-templates is essential to realize a diameter selective synthesis of BNNTs (diameter of $0.7 \pm 0.1 \mathrm{~nm}$ has expected for thin SWBNNTs by them)

In this work we have calculated our systems based on the various distribution diameters of SWBNNTs and SWCNTs. The effects of these doping on the electrostatic properties of the inner and outer tubes can also be calculated for any further comparison and discussion.

Monajjemi and coworkers have simulated a large range of the nanotube carbons and other molecules via a wide range of methods and basis sets $^{11-127}$

Zigzag BNNTs $(n, 0)$ are expected to have direct band gap. On the other hand, armchair BNNTs $(\mathrm{m}, \mathrm{m})$ will have indirect band gap ${ }^{128}$. Because of their large band gap of ( $\sim \mathrm{eV})$, experiments using BNNTs as the conduction channel for field-effect transistors (FETs) showed that BNNTs allowed transport through only the valence band.

Another important feature about the band gaps of BNNTs is that they are tunable by doping with carbon, radial deformation, or by applying a transverse electric field across the BNNT "so-called giant stark effect"129.

Theoretical band structure calculations suggested that SWBNNT can either be p-type or n-type semiconductors by controlling the composition of carbon into SWBNNTs. Carbon impurities on the boron sites result in electron carriers while on the nitrogen sites result in hole carriers ${ }^{130}$.

On the other hand, the band-gap modification by radial deformation in BNNTs was predicted through first-principles pseudo-potential densityfunctional calculations.

In zigzag BN nanotubes, radial deformations due to transverse pressures of about $10 \mathrm{GPa}$ decrease the direct band gap of BNNTs from 5 to $2 \mathrm{eV}$, allowing for optical applications in the visible range ${ }^{130}$. However, the band gaps of armchair BNNTs are found to be insensitive to radial deformations. Finally, theory indicates that the band gap of BNNTs can be reduced and even completely removed by the application of transverse electric field onto BNNTs and simultaneously probing the electronic properties.

In this study we exhibited that the piezoelectricity for BNNTs causes to increase the capacity of BNNTs @ X doped-CNTs capacitor compare to CNTs @ CNTs. This phenomenon theoretically originated from the deformation effect due to the rolling of the planar hexagonal $\mathrm{BN}$ networks to form tubular structures ${ }^{131}$. Nakhmanson and coworkers showed that BNNT could be excellent piezoelectric systems ${ }^{132}$. As an example of calculations, piezoelectric constant for different zigzag BNNTs was found to increase along with the decrease of the radius of BNNTs [132] Experimentally, Bai et al. have shown that under in situ elastic bending deformation at room temperature 
inside a 300-kV high-resolution transmission electron microscope, a normally electrically insulating MWBNNT may transform to a semiconductor ${ }^{133}$.

One of the main purposes in this study of explaining the capacitor model is concerning the interaction between SWCNTs and SWBNTs is both of electronic transport properties and energy analysis. Although there are many data and a novel method for electronic transport reports for the double-wall carbon nanotubes, for BNNTs @ WCNTs there are not significant works, spatially the subject of this work for BNNTs @ X-doped-WCNTs is completely new.

Based on previous works, we simulated our model in viewpoint of various diameters and chirality by considering the SWCNTs and SWBNNTs.

\section{The electronic structure}

The electron density has been defined as

$$
\rho(r)=\eta_{i}\left|\varphi_{i}(r)\right|^{2}=\sum_{i} \eta_{i}\left|\sum_{l} C_{l, i} \chi_{i}(r)\right|^{2}
$$

${ }^{134-136}$ Where $\eta i$ is occupation number of orbital (i), is orbital wave function, $\chi$ is basis function and $C$ is coefficient matrix, the element of $i_{\text {th }}$ row $j_{\text {th }}$ column corresponds to the expansion coefficient of orbital $j$ respect to basis function $i$. Atomic unit for electron density can be explicitly written as e/Bohr ${ }^{3}$.

$$
\begin{aligned}
& \nabla \rho(r)=\left[\left(\frac{\partial \rho(r)}{\partial(x)}\right)^{2}+\left(\frac{\partial \rho(r)}{\partial(y)}\right)^{2}+\left(\frac{\partial \rho(r)}{\partial(z)}\right)^{2}\right]^{1} \\
& \nabla^{2} \rho(r)=\frac{\partial^{2} \rho(r)}{\partial x^{2}}+\frac{\partial^{2} \rho(r)}{\partial y^{2}}+\frac{\partial^{2} \rho(r)}{\partial z^{2}}
\end{aligned}
$$

${ }^{134-136}$ The kinetic energy density is not uniquely defined, since the expected value of kinetic energy operator

$$
<\varphi\left|-\left(\frac{1}{2}\right) \nabla^{2}\right| \varphi>
$$

can be recovered by integrating kinetic energy density from alternative definitions. One of commonly used definition is:

$$
k(r)=-\frac{1}{2} \sum_{i} \eta_{i} \varphi_{i}^{*}(r) \nabla^{2} \varphi_{i}(r)
$$

Relative to $K(r)$, the local kinetic energy definition given below guarantee positivizes everywhere; hence the physical meaning is clearer and is more commonly used.

The Lagrangian kinetic energy density, " $G(r)$ " is also known as positive definite kinetic energy density.

$G(r)=\frac{1}{2} \sum_{i} \eta_{i} \mid \nabla\left(\left.\varphi_{i}\right|^{2}=\frac{1}{2} \sum_{i} \eta_{i}\left\{\left[\left(\frac{\partial \varphi_{i}(r)}{\partial(x)}\right)^{2}+\left(\frac{\partial \varphi_{i}(r)}{\partial(y)}\right)^{2}+\left(\frac{\partial \varphi_{i}(r)}{\partial(z)}\right)^{2}\right]\right\}\right.$

$K(r)$ and $G(r)$ are directly related by Laplacian of electron density

$\frac{1}{4} \nabla^{2} \rho(r)=G(r)-K(r)$

Becke and Edgecombe noted that spherically averaged likespin conditional pair probability has direct correlation with the Fermi hole and then suggested electron localization function $(E L F)^{135}$.

$$
E L F(r)=\frac{1}{1+\left[D(r) / D_{0(r)}\right]^{2}}
$$

where

$$
\mathrm{D}(\mathrm{r})=\frac{1}{2} \sum_{i} \eta_{i}\left|\nabla \varphi_{i}\right|^{2}-\frac{1}{8}\left[\frac{\left|\nabla_{\rho_{\alpha}}\right|^{2}}{\rho_{\alpha}(r)}+\frac{\left|\nabla_{\rho_{\beta}}\right|^{2}}{\beta(r)}\right]
$$

and

$$
D_{0(r)}=\frac{3}{10}\left(6 \pi^{2}\right)^{\frac{2}{3}}\left[\rho_{\alpha}(r)^{\frac{5}{3}}+\rho_{\beta}(r)^{\frac{5}{3}}\right]
$$

for close-shell system, since

$$
\rho_{a x}(r)=\rho_{\beta}(r)=\frac{1}{2} \rho, D \text { and } D 0 \text { terms can be simplified }
$$
as

$$
\mathrm{D}(\mathrm{r})=\frac{1}{2} \sum_{\mathrm{i}} \eta_{i}\left|\nabla \varphi_{i}\right|^{2}-\frac{1}{8}\left[\frac{|\nabla \rho|^{2}}{\rho(r)}\right]
$$


$D_{0(r)}=\frac{3}{10}\left(3 \pi^{2}\right)^{\frac{2}{3}} \rho(r)^{\frac{5}{3}}$

Savin et al. have reinterpreted ELF in the view of kinetic energy, which makes ELF also meaningful for Kohn-Sham DFT wave-function or even post-HF wave-function. They indicated that $D(\mathbf{r})$ reveals the excess kinetic energy density caused by Pauli repulsion, while $D O(r)$ can be considered as Thomas-Fermi kinetic energy density. Localized orbital locator (LOL) is another function for locating high localization regions likewise ELF, defined by Schmider and Becke in the paper. We have calculated the Hamiltonian kinetic energy and density Electron
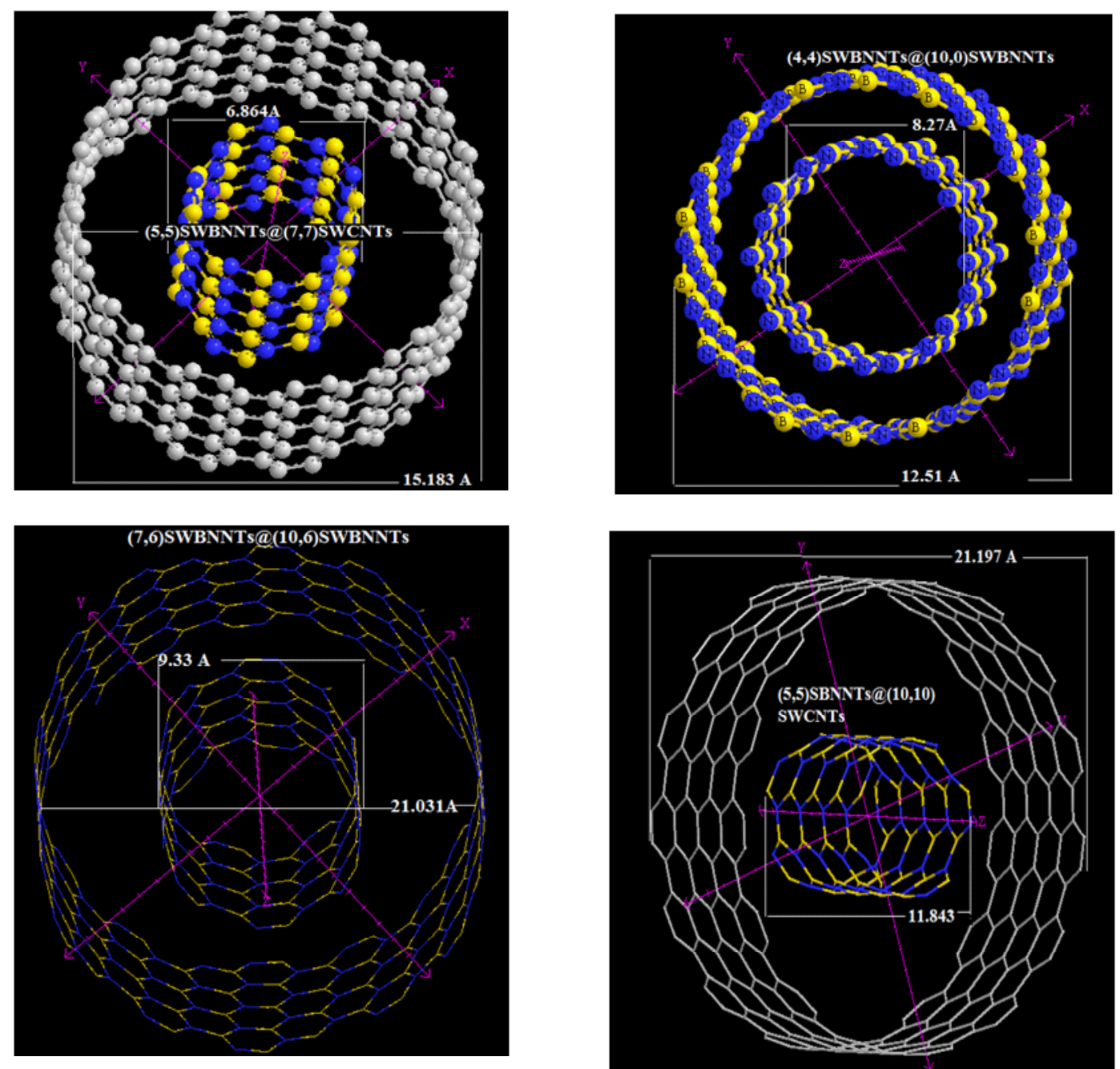

Fig.1: Various SWCNTs and SWBNNTs inside together to produce DWBNNTs \&DWCNTS

localization function of lithium in diffusion model for several of SWBNNTs, SWCNTs and WBNNTs@ SWCNTS

The Total electrostatic potential (ESP) measures the electrostatic interaction between a unit point charges placed at $r$ and the system of interest. A positive (negative) value implies that current position is dominated by nuclear (electronic) charges. Molecular electrostatic potential (ESP) has been widely used for prediction of nucleophilic and electrophilic sites for a long time.

It is also valuable in studying hydrogen bonds, halogen bonds, molecular recognitions and

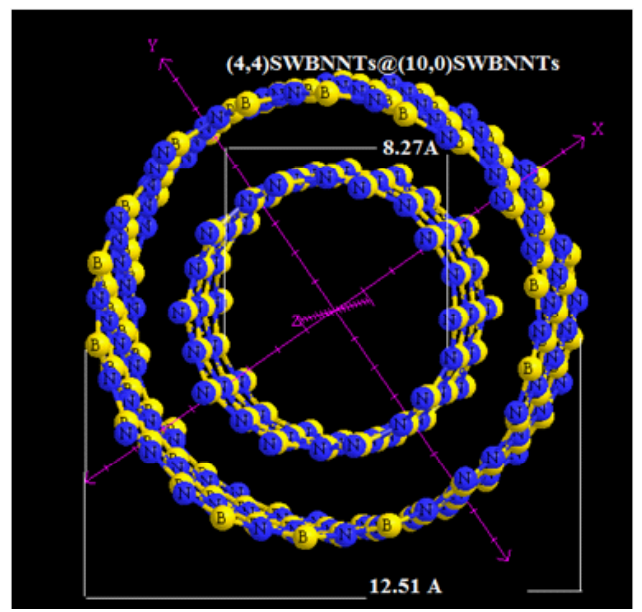


the intermolecular interaction of aromatics. The ESP evaluated under default value is accurate enough in general cases.

\section{Computational details}

Calculations were performed using Gaussian 09 and GAMESS-US packages ${ }^{137}$. In this study, we have mainly focused on getting the optimized results for each tube from HF and DFT methods including the B3lyp and Blyp. Extendedhuckel calculations using Gaussian program have done for the non-bonded

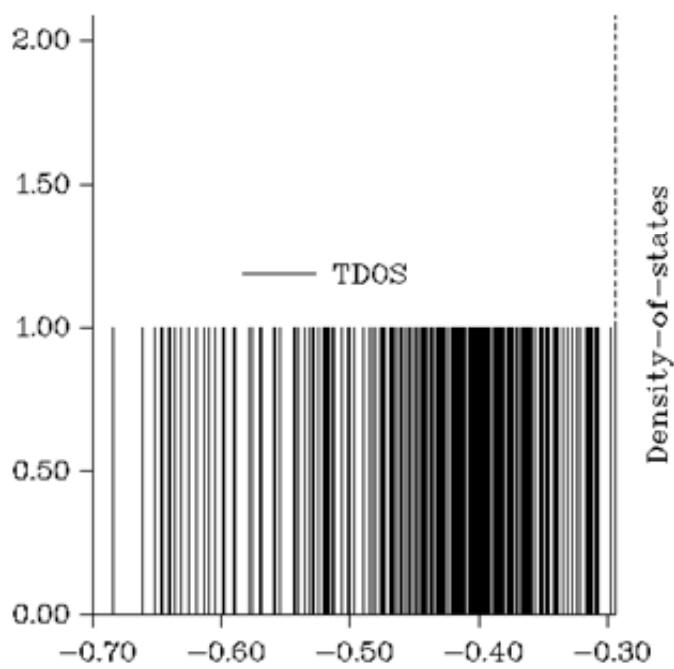

Fig. 2: Density of state for SW $(4,4)$ BNNTs@ $(10,10)$ SWBNNTs

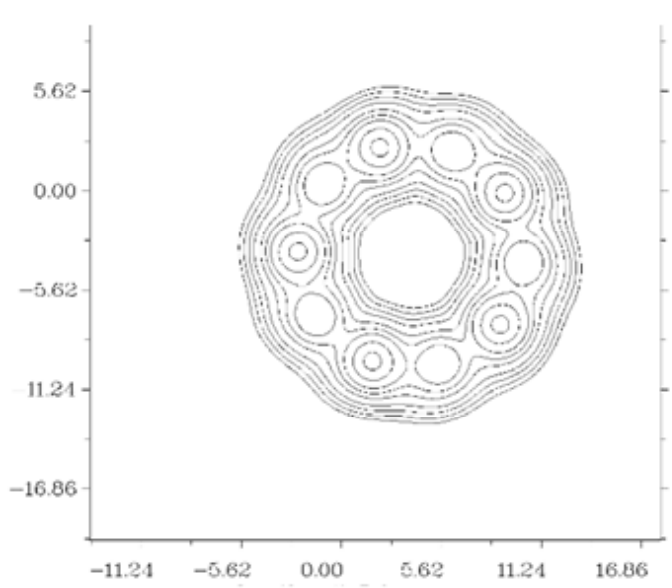

Fig. 3: Counter map of $(5,5)$ SWBNNTs interaction between BNNTs and X-doped-SWNTs which are monotonous through the comparison between different situations.

Geometry optimizations and electronic structure calculations for tubes have been carried out using the $6-31 \mathrm{~g}$ and $6-31 \mathrm{~g}^{*}$ basis sets.

A fixed BNNT geometry with B-N bond lengths of $1.445 \AA$, and $B-H$ and $\mathrm{N}-\mathrm{H}$ bond lengths of $1.185 \AA$ and $1.05 \AA$, respectively, are chosen with no further geometry optimization. The outer ring, initially placed at the center of the inner tube, is rigidly axially shifted and rotated around the fixed inner shell.

The charge transfer and electrostatic potential-derived charge were also calculated using the Merz-Kollman-Singh ${ }^{138}$, chelp ${ }^{139}$ or chelpG ${ }^{140}$ the charge calculation methods based on molecular electrostatic potential (MESP) fitting are not wellsuited for treating larger systems whereas some of the innermost atoms are located far away from the points at which the MESP is computed.

In such a condition, variations of the innermost atomic charges will not lead towards a significant change of the MESP outside of the molecule, meaning that the accurate values for the innermost atomic charges are not well-determined by MESP outside the molecule. This approach (CHELPG) is shown to be considerably less

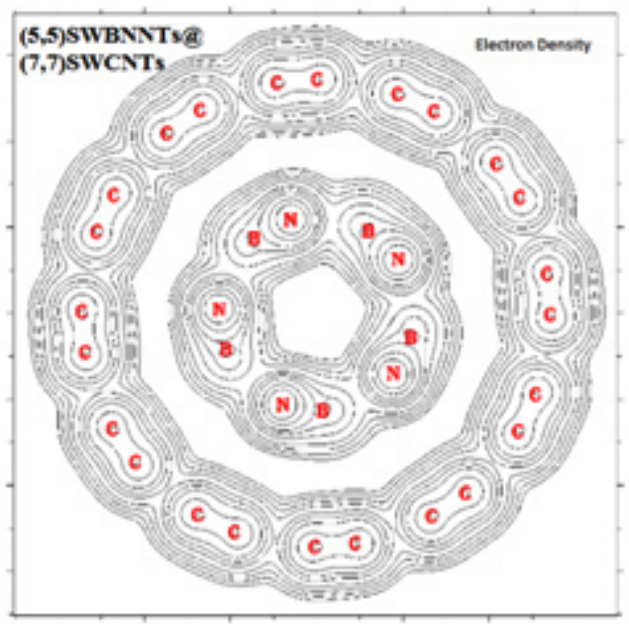

Fig. 4: Counter map of $(5,5)$ SWBNNTs@ $(7,7)$ SWCNNTs 
dependent upon molecular orientation than the original CHELP program. The results are compared to those obtained by using CHELP.

In the CHELPG (Charges from Electrostatic Potentials using a Grid based method), atomic charges are fitted to reproduce the molecular electrostatic potential (MESP at a number of points around the molecule. The MESP is calculated at a number of grid points spaced 3.0 pm apart and distributed regularly in a cube. Charges derived in this way don't necessarily reproduce the dipole moment of the molecule. CHELPG charges are frequently considered superior to Mulliken charges as they depend much less on the underlying theoretical method used to compute the wave function (and thus the MESP) $)^{141-142}$.

\section{RESULT AND DISCUSSION}

The results are shown in Figs 1-7 and tables1. The system has been modeled with Armchair, Zigzag and Chiral as an impurity among the atoms of the outer nanotube layer. The total energy contains three terms including the band structure energy of the inner and outer tubes and the electrostatic energy of the layers charge distribution. In this model, the SWBNNTs compare to SWCNTs tubes have been chosen by various diameters, therefore, as the SWNTCs, the boron atom also

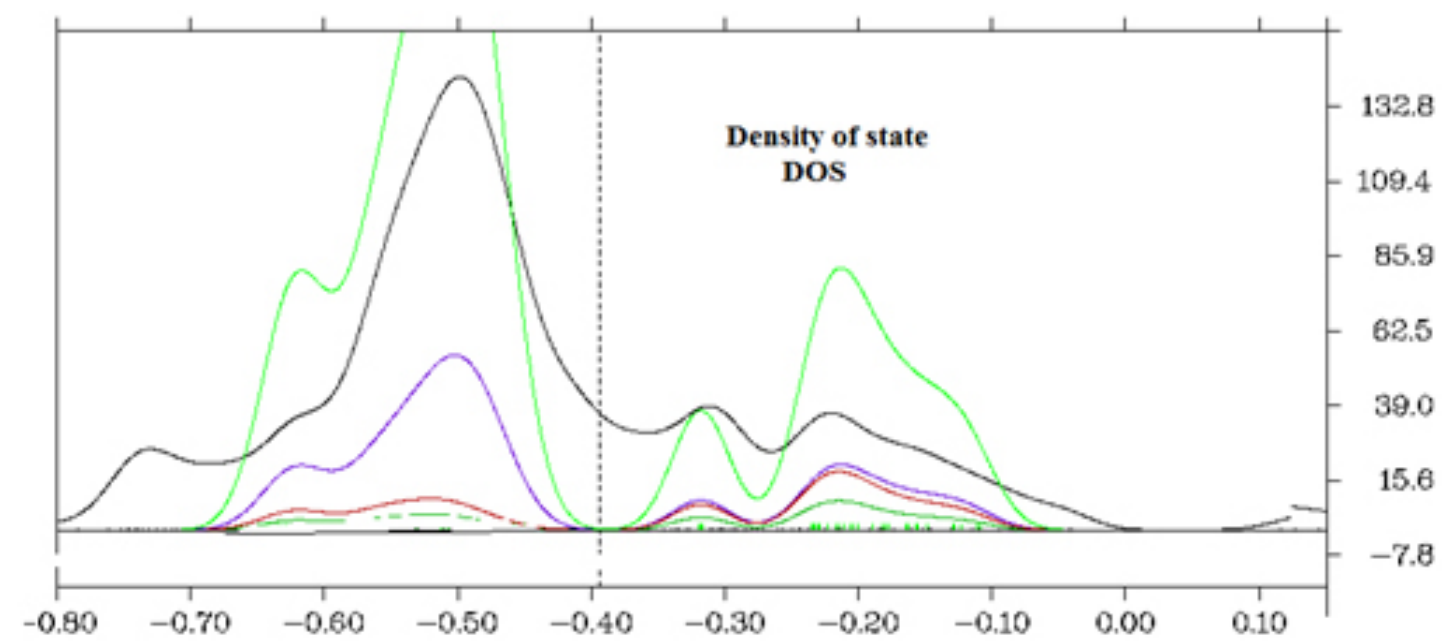

Fig. 5: Density of state

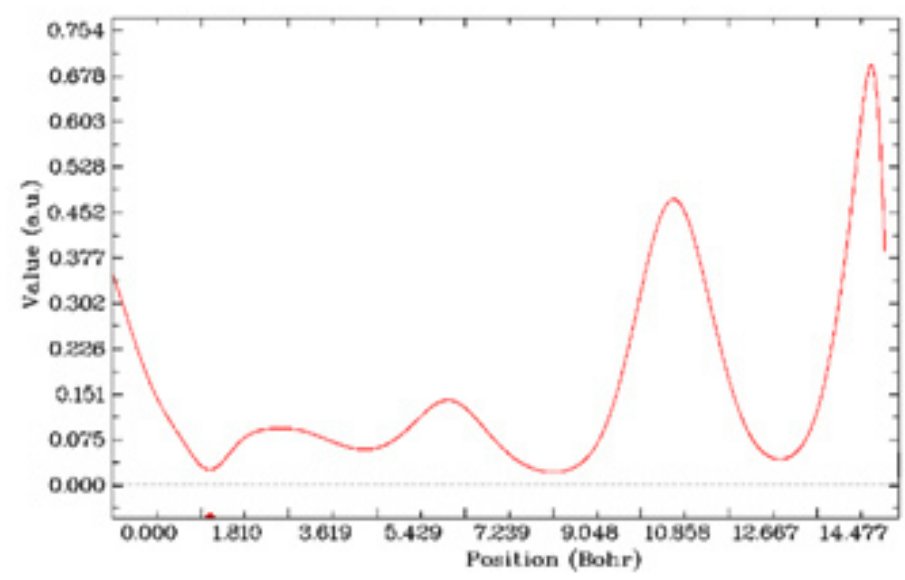

Fig. 6: The electron density between 0.0 to 15.0 (Bohr) position in $(10,10)$ SWCNTs 


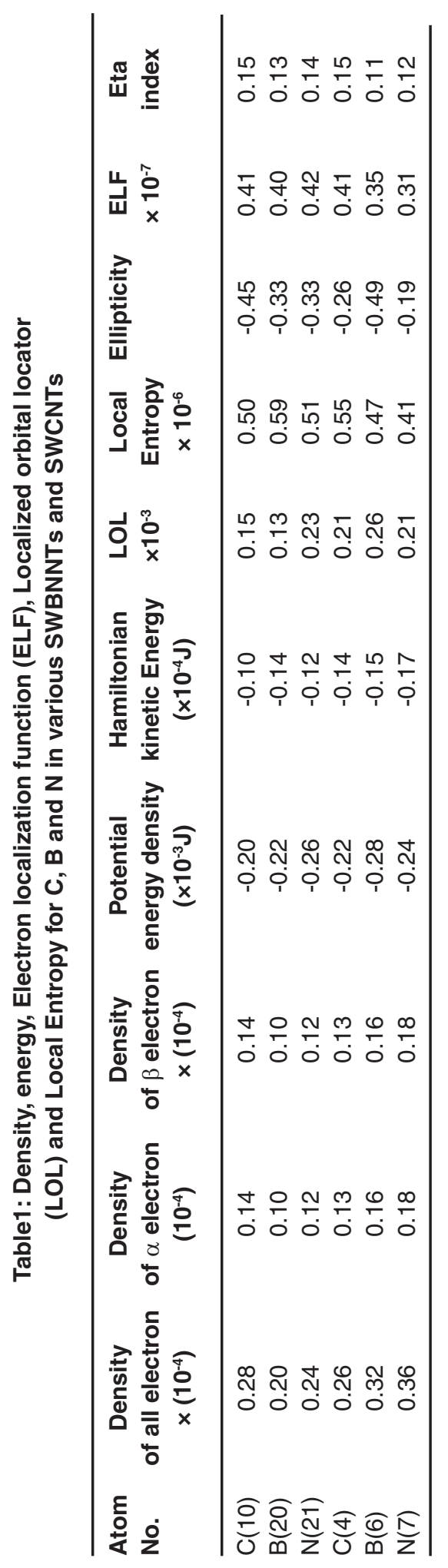

undergoes the $\mathrm{sp}^{2}$ hybridization. Due to the relatively similar size of $C$ and $B$, no significant distortion in 3-D structure of tube is expected, except for the change in adjoining the bond length. By doping the boron atoms in SWNTCs, the Fermi level shifts significantly below the Dirac point resulting in a p-type doping. This would break the symmetry of tubes into two sub layers due to presence of the B atoms which would eventually lead towards a change of the behavior of tubes from semimetal to conductor.

It is shown that the buckling rapidly increases with decreasing the tube diameter but is nearly independent of the chirality. This result is consistent with the reported trend revealed by tightbinding and ab initio calculations. The wall buckling is driven by different hybridizations related with the $\mathrm{B}$ and $\mathrm{N}$ atoms.

Two physical effects act in concert to segregate most of the holes onto the outer nanotube. First, the band gaps of the smaller diameter tubes tend to be larger, so they empty last. Second, the cylindrical geometry preferentially raises the electrostatic potential at the inner tube. Only the charge on the inner tube affects the potential difference between the two layers. Figures 1 shows the results of the energy minimization. The stability of small BNNTs encapsulated in a larger $\mathrm{x}$-doped CNT is confirmed by this work. As the total energies of the small BNNTs are higher than those of their uncurled states, examination the behavior of the small BNNTs inside a larger BNNT should be necessary. It is apparent that the small BNNTs become globally stable in their energy favorable outer shells. Encapsulation induced enhancement in stability should be able to extend into other small BNNTs. The electronic properties of SWCNTs and SWBNNTs are listed in table1.

We will call the lowest $\pi^{\star}$ state as singly degenerate state hereafter for all the small BNNTs. In BNNTs, the $\pi^{\star}$ states are mainly distributed on the $B$ atoms from the pz orbitals perpendicularly to the tube wall. So it can be imagined that a shorter $\mathrm{B}-\mathrm{B}$ distance along the circumference will be more favorable for an overlapping of the singly degenerate state inside the tube, thus enhancing the $\pi^{\star}-\sigma^{*}$ hybridization. With increasing chiral angle of the BNNT, there are two primary variations that could 
significantly affect such overlapping: (i) Increasing nearest B-B distance along the circumference.

Besides, we also note that the repulsion between the NFE and $\pi^{*}$ bands becomes increasingly significant from the $(2,2)$ to $(4,0)$ BNNTs, indicating that the NFE- $\pi^{*}$ hybridization becomes stronger with decreasing chiral angle as well. This phenomenon gives us a hint that the amplitude of NFE- $\pi$ hybridization is proportional to that of $\pi^{*}-\sigma^{*}$ hybridization. On the other hand, it is found that the nearest B-B distance along the circumference can also be reduced by decreasing the tube diameter, which is decreased to $2.16 \AA$ in the $(3,0)$ BNNT. That is why the energy gap displays apparent diameter dependence in the small zigzag BNNTs.

The data of Density of all electron, Density of electron, Density of $\beta$ electron, Potential energy density, Hamiltonian, kinetic energy, Hamiltonian kinetic Energy, LOL, local Entropy, ellipticity, ELF, eta index of several atoms in nanotube have listed in table1. For calculation the electron spin density from the difference between alpha and beta density, we have used $\rho^{s}(r)=\rho^{\alpha}(r)-\rho^{\beta}(r)$ then the spin polarization parameter function will be returned instead of spin density $\xi(r)=\frac{\rho^{\alpha}(r)-\rho^{\beta}(r)}{\rho^{\alpha}(r)+\rho^{\beta}(r)} \quad$.The kinetic energy density, Lagrangian kinetic energy density, and the electrostatic potential from nuclear / atomic charges can be calculated as the 1-5 eqs.

The larger the electron localization is in a region, the more likely the electron motion is confined within it. If electrons are completely localized, then they can be distinguished from the ones outside. Bader found that the regions which have large electron localization must have large magnitudes of Fermi hole integration. However, the Fermi hole is a six-dimension function and thus difficult to be studied visually.

Since $D 0(\mathbf{r})$ from eqs. $5-10$ is introduced into ELF as reference, what the ELF reveals is actually a relative localization. ELF is within the range of $[0,1]$. A large ELF value means that electrons are greatly localized, indicating that there is a covalent bond, a lone pair or inner shells of the atom involved. ELF has been widely used for a wide variety of systems, such as organic and inorganic small molecules, atomic crystals, coordination compounds, clusters, and for different problems, such as the revealing atomic shell structure, classification of chemical bonding, verification of charge-shift bond, studying aromaticity.

LOL has similar expression compared to ELF. Actually, the chemically significant regions that highlighted by LOL and ELF are generally qualitative comparable, while Jacobsen pointed out that LOL conveys more decisive and clearer picture than ELF, Obviously LOL can be interpreted in kinetic energy way as for ELF; however LOL can also be interpreted in view of localized orbital. Small (large) LOL value usually appears in boundary (inner) region of localized orbitals because the gradient of orbital wave-function is large (small) in this area. The value range of LOL is identical to ELF, namely $[0,1]$

\section{REFERENCES}

1. M. Topsakal, S. Ciraci, Phys. Rev. B 85, 045121. (18-20) (2012)

2. Dai, H. J.; Rinzler, A. G.; Nikolaev, P.; Thess, A.; Colbert, D. T.; Smalley, R. E. Chem. Phys. Lett. 260, 471, (1996)

3. Sinnott, S. B.; Andrews, R.; Qian, D.; Rao, A. M.; Mao, Z.; Dickey, E. C.; Derbyshire, F. Chem. Phys. Lett. 315, 25, (1999)

4. Chin Li Cheung, Andrea Kurtz, Hongkun Park, and Charles M. Lieber, Diameter-Controlled Synthesis of Carbon Nanotubes, J. Phys.
Chem. B, 106, 2429 2433, (2002)

5. Z. M. Li, Z. K. Tang, H. J. Liu, N. Wang, C. T. Chan, R. Saito, S. Okada, G. D. Li J. S. Chen, N. Nagasawa, and S. Tsuda, Phys. Rev. Lett. 87, 127401 (2001)

6. A. Rubio, J. L. Corkill, and M. L. Cohen, Phys. Rev. B 49, 5801 (1994).

7. S. Okada, S. Saito, and A. Oshiyama, Phys. Rev. B 65, 165410 (2002).

8. Xiang, H., Yang, J., Hou, J. \& Zhu, Q. Phys. Rev. B 68, 035427 (2003). 
9. Chen, Y., Zou, J., Campbell, S. J. \& Le Caer, G. Appl. Phys. Lett. 84, 2430-2432, (2004)

10. Kitaura, R. et al. Angew. Chem. Int. Ed. 48, 8298-8302, (2009)

11. Monajjemi, M.; Lee, V.S.; Khaleghian, M.; B. Honarparvar, B.; F. Mollaamin, F. J. Phys. Chem C. 2010, 114, 15315

12. Monajjemi, M. Struct Chem. 2012, 23,551580

13. Monajjemi, M.; Chegini, H.; Mollaamin, F.; Farahani, P. Fullerenes, Nanotubes, and Carbon Nanostructures. 2011, 19, 469-482

14. Monajjemi, M .; Afsharnezhad ,S.; Jaafari , M.R.; Abdolahi ,T.; Nikosade ,A.; Monajemi ,H.; Russian Journal of physical chemistry A, 2007, 2,1956-1963

15. Monajjemi, M.; Baei, M.T.; Mollaamin, F. Russian Journal of Inorganic Chemistry. 2008, 53 (9), 1430-1437

16. Monajjemi, M.; Rajaeian, E.; Mollaamin, F.; Naderi, F.; Saki, S. Physics and Chemistry of Liquids. 2008, 46 (3), 299-306

17. Monajjemi, M.; Boggs, J.E. J. Phys. Chem. A, 2013, 117, 1670 "1684

18. Mollaamin, F.; Monajjemi, M, Journal of Computational and Theoretical Nanoscience. 2012, 9 (4) 597-601

19. Monajjemi, M.; Khaleghian, M, Journal of Cluster Science. 2011, 22 (4) , 673-692

20. Mollaamin, F.; Varmaghani, Z.; Monajjemi, M, Physics and Chemistry of Liquids. 2011, 49 318

21. Nafisi, S.; Monajemi, M.; Ebrahimi, S. Journal of Molecular Structure. 2004,705 (3) 35-39

22. Fazaeli, R.; Monajjemi, M.; Ataherian, F.; Zare, K. Journal of Molecular Structure: THEOCHEM.2002, 581 (1), 51-58

23. Monajjemi, M.; Razavian, M.H.; Mollaamin,F.; Naderi,F.; Honarparvar,B.; Russian Journal of Physical Chemistry A , 2008 , 82 (13), 22772285

24. Monajjemi, M.; Seyed Hosseini, M.; Mollaamin, F. Fullerenes, Nanotubes, and Carbon Nanostructures. 2013, 21, 381-393

25. Monajjemi, M.; Faham, R.; Mollaamin, F. Fullerenes, Nanotubes, and Carbon Nanostructures, 2012 20, 163-169

26. Mollaamin, F.; Najafi, F.; Khaleghian, M.;
Khalili Hadad, B.; Monajjemi, M. Fullerenes, Nanotubes, and Carbon Nanostructures, 2011 19, 653-667

27. Mollaamin, F.; Baei, MT.; Monajjemi, M.; Zhiani, R.; Honarparvar, B.; Russian Journal of Physical Chemistry A, Focus on Chemistry, 2008, 82 (13), 2354-2361

28. Monajjemi, M. Chemical Physics. 2013, 425, 29-45

29. Monajjemi, M.; Heshmat, M.; Aghaei, H.; Ahmadi, R.; Zare, K. Bulletin of the Chemical Society of Ethiopia, 2007, 21 (1)

30. Monajjemi, M.; Honarparvar, B. H. ; Haeri, H. ; Heshmat ,M.; Russian Journal of Physical Chemistry C. 2006, 80(1):S40-S44

31. Monajjemi, M.; Ketabi, S.; Amiri, A. Russian Journal of Physical Chemistry, 2006, 80 (1), S55-S62

32. Yahyaei, H.; Monajjemi, M.; Aghaie, H.; K. Zare, K. Journal of Computational and Theoretical Nanoscience. 2013, 10, 10, 2332-2341

33. Mollaamin, F.; Gharibe, S.; Monajjemi, M. Int. J. Phy. Sci, 2011, 6, 1496-1500

34. Monajjemi, M.; Ghiasi, R.; Seyed Sadjadi, M.A. Applied Organometallic Chemistry,2003, 17, 8, 635-640

35. Monajjemi, M.; Wayne Jr, Robert. Boggs, J.E. Chemical Physics. 2014, 433, 1-11

36. Monajjemi, M.; Sobhanmanesh, A.; Mollaamin, F. Fullerenes, Nanotubes, and Carbon Nanostructures, 2013, 21 47-63

37. Monajjemi, M.; Mollaamin, F. Journal of Computational and Theoretical Nanoscience, 2012, 9 (12) 2208-2214

38. Monajjemi, M.; Honarparvar, B.; Nasseri, S. M. .; Khaleghian M. Journal of Structural Chemistry. 2009, 50, 1, 67-77

39. Monajjemi, M.; Aghaie, H.; Naderi, F. Biochemistry (Moscow).2007, 72 (6), 652657

40. Ardalan, T.; Ardalan, P.; Monajjemi, M. Fullerenes, Nanotubes, and Carbon Nanostructures, 2014, 22: 687-708

41. Mollaamin, F.; Monajjemi, M.; Mehrzad, J. Fullerenes, Nanotubes, and Carbon Nanostructures. 2014, 22: 738-751

42. Monajjemi, M.; Najafpour, J.; Mollaamin, 
F. Fullerenes, Nanotubes, and Carbon Nanostructures. 2013, 21(3), 213-232

43. Monajjemi, M.; Karachi, N.; Mollaamin, F. Fullerenes, Nanotubes, and Carbon Nanostructures, 2014, 22: 643-662

44. Yahyaei, H.; Monajjemi, M. Fullerenes, Nanotubes, and Carbon Nanostructures.2014, 22(4), 346-361

45. Monajjemi, M. Falahati, M.; Mollaamin, F.; lonics, 2013, 19, 155-164

46. Monajjemi, M.; Mollaamin, F. Journal of Cluster Science, 2012, 23(2), 259-272

47. Tahan, A.; Monajjemi, M. Acta Biotheor, 2011, 59, 291-312

48. Lee, V.S.; Nimmanpipug, P.; Mollaamin, F.; Kungwan, N.; Thanasanvorakun, S..; Monajjemi, M. Russian Journal of Physical Chemistry A, 2009, 83, 13, 2288-2296

49. Monajjemi, M.; Heshmat, M.; Haeri, HH, Biochemistry (Moscow), 2006, 71 (1), S113S122

50. Monajjemi, M.; Yamola, H.; Mollaamin, F. Fullerenes, Nanotubes, and Carbon Nanostructures, 2014, 22, 595-603

51. Mollaamin, F.; Layali, I.; Ilkhani A. R.; Monajjemi, M. African Journal of Microbiology Research .2010, 4(24) 2795-2803

52. Mollaamin, F.; Shahani poor, p K. .; Nejadsattari, T.; Monajjemi, M. African Journal of Microbiology Research. 2010, 4(20) 20982108

53. Monaijemi, M.; Ahmadianarog, M. Journal of Computational and Theoretical Nanoscience. 2014, 11(6), 1465-1471

54. Monajjemi, M.; Jafari Azan, M.; Mollaamin, F. Fullerenes, Nanotubes, and Carbon Nanostructures.2013, 21(6), 503-515

55. Mollaamin, F.; Monajjemi, M. Physics and Chemistry of Liquids .2012, 50, 5, 2012, 596-604

56. Monajjemi, M.; Khosravi, M.; Honarparvar, B.; Mollaamin, F.; International Journal of Quantum Chemistry, 2011, 111, 2771-2777

57. Khaleghian, M.; Zahmatkesh, M.; Mollaamin, F.; Monajjemi, M. Fullerenes, Nanotubes, and Carbon Nanostructures, 2011, 19(4): 251-261

58. Monajjemi, M.; Baheri, H.; Mollaamin, F. Journal of Structural Chemistry.2011 52(1), 54-59
59. Mahdavian, L.; Monajjemi, M.; Mangkorntong, N. Fullerenes, Nanotubes and Carbon Nanostructures, 2009, 17 (5), 484-495

60. Monaijemi, M., Mahdavian, L., Mollaamin, F. Bull. Chem. Soc. Ethiop. 2008, 22(2), 277286

61. Monajjemi, M.; Afsharnezhad, S, Jaafari, M.R..; Mirdamadi, S..; Mollaamin, F..; Monajemi, $\mathrm{H}$. Chemistry .2008, 17 (1), 55-69

62. Monajjemi, M.; Mollaamin, F.; Gholami, M. R.; Yoozbashizadeh, H.; Sadrnezhaad, S.K.; Passdar, H.; Main Group Metal Chemistry, 2003, 26, 6, 349-361

63. Monajjemi, M.; Azad ,MT.; Haeri, HH.; Zare, K.; Hamedani, Sh.; JOURNAL OF CHEMICAL RESEARCH-S.2003, (8): 454-456

64. Monajjemi, M.; Najafpour, J. Fullerenes, Nanotubes, and Carbon Nanostructures, 2014, 22(6): 575-594

65. Monajjemi, M.; Noei, M.; Mollaamin, F. Nucleosides, Nucleotides and Nucleic Acids. 2010 29(9):676-683

66. Ghiasi, R.; Monajjemi, M. Journal of Sulfur Chemistry .2007, 28, 5, 505-511

67. Monajjemi, M.; Ghiasi, R.; Abedi, A. Russian Journal of Inorganic Chemistry.2005, 50(3), 382-388

68. Monajjemi, M. .; Naderi, F.; Mollaamin, F.; Khaleghian, M. J. Mex. Chem. Soc. 2012, 56(2), 207-211

69. Monajjemi, M.; Farahani, N.; Mollaamin, F. Physics and Chemistry of Liquids, 2012, 50(2) 161-172

70. Monajjemi, M.; Seyed Hosseini, M. Journal of Computational and Theoretical Nanoscience .2013, 10 (10), 2473-2477

71. Monajjemi , M.; Honaparvar, B.; Khalili Hadad ,B.; Ilkhani ,AR.; Mollaamin, F. African Journal of Pharmacy and Pharmacology .2010, 4(8), $521-529$

72. Monajjemi, M. Theor Chem Acc, 2015, 134:77 DOI 10.1007/s00214-015-1668-9

73. Monajjemi, M. Journal of Molecular Modeling , 2014, 20, 2507

74. Monajjemi, M.; Honarparvar, B.; Monajemi, H.;. Journal of the Mexican Chemical Society, 2006, 50 (4), 143-148

75. Monajjemi, M.; Khaleghian, M.; Mollaamin, F. Molecular Simulation. 2010, 36, 11, 865-

76. Ilkhani, Ali R.; Monajjemi, M. Computational 
and Theoretical Chemistry.2015 1074, 19-25

77. Monajjemi, M. Biophysical Chemistry. 2015 207,114-127

78. Monajjemi, M., Moniri, E., Panahi, H.A , Journal of Chemical and Engineering Data.2001, 1249-1254.

79. Mollaamin, F.; Najafpour, J.; Ghadami, S.; Ilkhani, A. R.; Akrami, M. S.; Monajjemi, M. Journal of Computational and Theoretical Nanoscience. 11 (5), 1290-1298

80. Monajjemi, M.; Ghiasi, R.; Ketabi, S.; Passdar, H.; Mollaamin, F. Journal of Chemical Research . 2004, 1, 11.

81. Monajjemi, M.; Heshmat, M.; Haeri, H.H. Biochemistry (Moscow).2006, 71, 113-122

82. Monajjemi, M.; Heshmat, M.; Aghaei, H.;Ahmadi, R.; Zare, K. Bulletin of the Chemical Society of Ethiopia. 2007, 21, 111-116

83. Monajjemi, M., Kharghanian, L., Khaleghian, M., Chegini, H. Fullerenes Nanotubes and Carbon Nanostructures.2014, 22, 8, 0.1080/1536383X.2012.717563

84. Sarasia, E.M.; Afsharnezhad, S.; Honarparvar, B.; Mollaamin, F.; Monajjemi, M.Physics and Chemistry of Liquids. 2011, 49 (5), 561-571

85. Amiri, A.; Babaeie, F.; Monajjemi, M. Physics and Chemistry of Liquids. 2008, 46, 4, 379389

86. Monajjemi, M.; Heshmat, M.; Haeri, H.H.; Kaveh, F. Russian Journal of Physical Chemistry A, 2006, 80, 7, 1061-1068

87. Monajjemi, M.; Moniri, E.; Azizi, Z.; Ahmad Panahi, H. Russian Journal of Inorganic Chemistry. 2005, 50, 1, 40-44

88. Jalilian,H.; Monajjemi, M. Japanese Journal of Applied Physics. 2015, 54, 8, 08510

89. Mollaamin, F.; Monajjemi, M. Journal of Computational and Theoretical Nanoscience. 2015, 12, 6, 1030-1039

90. Felegari, Z.; Monajjemi, M. Journal of Theoretical and Computational Chemistry. 2015, 14, 3, 1550021

91. Shojaee, S., Monajjemi, M. Journal of Computational and Theoretical Nanoscience. 2015, 12, 3, 449-458

92. Esmkhani, R.; Monajjemi, M. Journal of Computational and Theoretical Nanoscience. 2015. 12, 4, 652-659
93. Monajjemi, M., Seyedhosseini, M., Mousavi, M., Jamali, Z. Journal of Computational and Theoretical Nanoscience. 2015 , 23 (3), 239244

94. Ghiasi, R.; Monajjemi, M.; Mokarram, E.E.; Makkipour, P. Journal of Structural Chemistry. 2008, 4 , 4, 600-605

95. Mahdavian, L.; Monajjemi, M. Microelectronics Journal. 2010, 41(2-3), 142-149

96. Monajjemi, M.; Baie, M.T.; Mollaamin, F. Russian Chemical Bulletin.2010, 59, 5, 886889

97. Bakhshi, K.; Mollaamin, F.; Monajjemi, M. Journal of Computational and Theoretical Nanoscience. 2011, 8, 4, 763-768

98. Darouie, M.; Afshar, S.; Zare, K., Monajjemi, M. journal of Experimental Nanoscience.2013, 8, 4, 451-461

99. Amiri, A.; Monajjemi, M.; Zare, K.; Ketabi, S. Physics and Chemistry of Liquids. 2006, 44, 4, 449-456.

100. Zonouzi, R.;Khajeh, K.; Monajjemi, M.; Ghaemi, N. Journal of Microbiology and Biotechnology. 2013, 23, 1 ,7-14

101. Ali R. Ilkhani .; Majid Monajjemi, Computational and Theoretical Chemistry.2015, 1074 19-25

102. Tahan, A.; Mollaamin, F.; Monajjemi, M. Russian Journal of Physical Chemistry $A$, 2009, 83 (4), 587-597

103. Khalili Hadad, B.; Mollaamin, F.; Monajjemi, M, Russian Chemical Bulletin,2011, 60(2):233236

104. Mollaamin, F.; Monajjemi, M.; Salemi, S.; Baei, M.T. Fullerenes Nanotubes and Carbon Nanostructures, 2011, 19, 3, 182-196

105. Mollaamin, F.; Shahani Pour.; K., Shahani Pour, K.; ilkhani, A.R.; Sheckari, Z., Monajjemi, M Russian Chemical Bulletin, 2012 , 61(12), 2193-2198

106. Shoaei, S.M.; Aghaei, H.; Monajjemi, M.; Aghaei, M. Phosphorus, Sulfur and Silicon and the Related Elements. 2014, 189, 5; 652660

107. Mehrzad, J., Monajjemi, M., Hashemi, M , Biochemistry (Moscow).2014, 79 (1), 31-36

108. Moghaddam, N.A., Zadeh, M.S., Monajjemi, M. Journal of Computational and Theoretical Nanoscience , 2015 , Vol. 12, No. 3, doi:10.1166/jctn.2015.3736 
109. Joohari, S.; Monajjemi, M , Songklanakarin Journal of Science and Technology , 2015 , 37(3):327

110. Rajaian, E., Monajjemi, M., Gholami, M.R, Journal of Chemical Research - Part S,2002, $6,1,279-281$

111. Ghassemzadeh, L., Monajjemi, M., Zare, K, Journal of Chemical Research - Part S, 2003, 4, 195-199

112. Maryam Mehdizadeh Barforushi, Soodeh Safari, and Majid Monajjemi J. Comput. Theor. Nanosci. 12, 3058-3065 (2015)

113. Fatemeh Mollaamin, Alireza Ilkhani, Neda Sakhaei, Behnaz Bonsakhteh, Afsaneh Faridchehr, Sogand Tohidi, and Majid Monajjemi J. Comput. Theor. Nanosci., 2015; 12, 3148-3154.

114. Hassan Rahmati and Majid Monajjemi J. Comput. Theor. Nanosci. 2015; 12, 34733481.

115. Roghieh Tarlani Bashiz and Majid Monajjemi J. Comput. Theor. Nanosci., 2015; 12, 38083816.

116. Akbar Mehrabi Nejad and Majid Monajjemi J. Comput. Theor. Nanosci. 2015; 12, 39023910

117. Majid Monajjemi *, Samira Bagheri, Matin S. Moosavi, Nahid Moradiyeh, Mina Zakeri, Naime Attarikhasraghi, Nastaran Saghayimarouf, Ghorban Niyatzadeh, Marzie Shekarkhand, Mohammad S. Khalilimofrad, Hashem Ahmadin and Maryam Ahadi, Molecules 2015, 20, 21636-21657; doi:10.3390/ molecules201219769

118. Elham Shabanzadeh and Majid Monajjemi, J. Comput. Theor. Nanosci, 2015; 12, 40764086

119. Akbar Elsagh, Hamidreza Jalilian, Elham Kianpour, Hediyeh Sadat Ghazi Mokri, Mostafa Rajabzadeh, Matin Sadat Moosavi, Fateme Ghaemi Amiri, and Majid Monajjemi J. Comput. Theor. Nanosci., 2015; 12, 42114218

120. Afsaneh Faridchehr, Abdolhossein Rustaiyan, and Majid Monajjemi J. Comput. Theor. Nanosci. 2015; 12, 4301-4314.

121. Sogand Tohidi, Majid Monajjemi, and Abdolhossein RustaiyanJ. Comput. Theor. Nanosci. 2015; 12, 4345-4351.

122. Mahsa Ali Akbari Zadeh, Hadi Lari, Leyla
Kharghanian, Ebrahim Balali, Ramona Khadivi, Hooriye Yahyaei, Fatemeh Mollaamin, and Majid Monajjemi J. Comput. Theor. Nanosci. 2015; 12, 4358-4367

123. Sahar Dezfooli, Hadi Lari, Ebrahim Balali, Ramona Khadivi, Farand Farzi, Nahid Moradiyeh, and Majid Monajjemi J. Comput. Theor. Nanosci. 2015; 12, 4478-4488.

124. Hamidreza Jalilian, Masoumeh Sayadian, Akbar Elsagh, Farand Farzi, Nahid Moradiyeh, Neda Samiei Soofi, Somayeh Khosravi, Nayyer T. Mohammadian, and Majid Monajjemi J. Comput. Theor. Nanosci. 2015; 12, 47854793.

125. Farand Farzi, Samira Bagheri, Mostafa Rajabzadeh, Masoumeh Sayadian, Hamidreza Jalilian, Nahid Moradiyeh, and Majid Monajjemi J. Comput. Theor. Nanosci. 2015; 12, 4862-4872.

126. Majid Monajjemi and Nayyer T. Mohammadian J. Comput. Theor. Nanosci. 2015; 12, 48954914.

127. Monajjemi, M., Chahkandi, B. Journal of Molecular Structure: Theochem, 2005, 714 (1), 28, 43-60.

128. A. Loiseau, F. Willaime, N. Demoncy, G. Hug and H. Pascard, Phys. Rev. Lett. 1996; 76; 4737.

129. K. H. Khoo, M. S. C. Mazzoni and S. G. Louie, Phys. Rev. B, 2004; 69, 201401R.

130. Y. Miyamoto, A. Rubio, M.L. Cohen and S. G. Louie, Phys. Rev. B, 1994; 50; 4976.

131. E. J. Mele and P. Kral, Phys. Rev. Lett. 2002; 88, 56803.

132. S. M. Nakhmanson, A. Calzolari,V. Meunier, J. Bernholc and M. B. Nardelli Phys. Rev. B, 2003; 67, 235406.

133. X. Bai, D. Golberg, Y. Bando, C. Zhi, C. Tang, M. Mitome and K. Kurashima, Nano Lett. 1997; 7632.

134. Lu T, Chen F, Acta Chim. Sinica, 2011; 69, 2393-2406

135. Lu T, Chen F, J. Mol. Graph. Model, 2012; 38: 314-323

136. Lu T, Chen F. Multiwfn: A Multifunctional Wavefunction Analyzer, J. Comp. Chem., 2012; 33; 580-592

137. M.W.Schmidt, K.K.Baldridge, J.A.Boatz, S.T.Elbert, M.S.Gordon, J.H.Jensen, S.Koseki, N.Matsunaga, K.A.Nguyen et al, 2004; 14(11); 
1347-1363.

138. L.E. Chirlian , M.M. Francl, J.comp.chem., 1987; 8,894

139. Brneman GM, Wiberg KB, J. Comp Chem, 1990; 11: 361.

140. Martin F, Zipse H Charge distribution in the water molecule-a comparison of methods. J Comp Chem., 2005; 26: 97 - 105

141. Naghsh,F, orient.j.chem, 2015, 31(1).,465478

142. Chitsazan, A, orient.j.chem, 2015, 31(1).,393408 